\title{
NGHIÊN CỨU MÔ HÌNH PHÁT HIỆN RÁC THẢI NHỰA VEN BIỄN SỬ DỤNG ẢNH MÁY BAY KHÔNG NGƯỜI LÁI VÀ MẠNG NƠ-RON TÍCH CHẬP SÂU
}

\author{
ĐÕ̃ TH!̣ NHUNG(1), NGUYẼ̃N TH!̣ DIẼ̃M MY(1), PHẠM VĂN MẠNH ${ }^{(1, *)}$, \\ PHẠM VŨ ĐÔNG(1), BÙI QUANG THÀNH( ${ }^{(1)}$, NGHIÊM VĂN TUẤN( ${ }^{(2)}$, \\ PHẠM MINH HẢI ${ }^{(3)}$ \\ ${ }^{(1)}$ Trường Đại học Khoa học Tự nhiên, Đại học Quốc gia Hà Nội \\ ${ }^{(2)}$ Cục Viễn thám Quốc gia, Bộ Tài nguyên và Môi truờng \\ ${ }^{(3)}$ Viện Khoa họ Đo đạc và Bản đồ, Bộ Tài nguyên và Môi trường
}

\section{Tóm tắt:}

Rác thải nhựa tại các khu vục ven biển có nguồn gốc do các hoạt động của con người và đang trở thành mối nguy cho môi truờng biển bởi số lượng lớn và khó phân hủy. Chúng đe dọa đến hệ động vật biển và phá hủy hệ sinh thái biển, gây ô nhiếm môi truờng và làm giảm giá trị cảnh quan của bãi biển. Các chuơng trình giám sát và các biện pháp giảm thiểu đã được đura ra để giải quyết vấn đề này trên toàn thế giới, với sự hỗ trọ̆ ngày càng nhiều của công nghệ hiện đại và tụ động hóa các quá trình phân tích. Hình ảnh tù máy bay không ngườ lái (UAV) và mạng no-ron tích chập sâu (DCNN) có thể được sử dụng hiệu quả để phát hiện, xác định và giám sát loại rác thải nhựa ven biển. Nghiên cứu này đề xuất một thuật toán phát hiện rác thải nhựa ven biển dựa trên cách tiếp cận mô hình DCNN có khả năng học tù dũ liệu không có cấu trúc hoạc không được gắn nhãn. Mô hình hoc máy dựa trên mạng nơ-ron tích chập sâu đã được đào tạo và thử nghiệm bằng cách sủ dụng 95 hình ảnh được chup tù Phantom 4 Pro với camera loại CMOS 1 inch có độ phân giải 20MP khu vục ven biển Hội An (Quảng Nam). Kết quả cho thấy, độ chính xác trong phân loại hình ảnh rác thải nhỵa ven biển và xác nhận chéo là̀n luợt là 0,87 và 0,83 . Nghiên cứu nhằm cung cấp một cách tiếp cận mói cho các nhà nghiên cứu, nhà quản lý vùng ven biển có ý định sử dụng ảnh UAV để giám sát và đánh giá mối đe dọa môi truờng tù các mảnh rác thải biển. Tuy nhiên, việc giám sát tụ động vẫn là một thách thức về công nghệ và cần có nhũ̃ng nghiên cứu thêm để cải thiện độ chính xác của các thuật toán hiện tại trong twoong lai.

Tù khóa: Rác thải nhựa, Viễn thám, UAV, Mạng nơ-ron tích chập, Học sâu.

\section{Tính cấp thiết}

Rác thải nhựa là một trong những thách thức môi trường lớn nhất hiện thế giới đang phải đối mặt [1]. Ô nhiễm rác thải nhựa hiện nay đang trở thành vấn đề nghiêm trọng đe dọa ngày càng lớn với môi trường trên toàn cầu, các mảnh vụn nhựa có tác động tiêu cực rất lớn đến môi trường và nơi cư trú của động thực vật [2]. Rác thải nhựa không phân huỷ thành các chất vô hại mà quá trình phân huỷ lại rất chậm và tồn tại lâu dài trong môi trường tự nhiên, gây ô nhiễm đại dương, đầu độc đất đai và giết chết động thực vật [3]. Rác thải nhựa có thể được vận chuyển từ trong đất liền ra đến vùng ven biển từ đó có thể xâm nhập vào đại dương thông qua hệ thống sông ngòi. Sự tồn tại của nhựa làm thay đổi chuyển động của nước và sự truyền nhiệt trên bãi biển, "rác biển" chiếm chỗ của các loài cần môi

Ngày nhận bài: 1/9/2021, ngày chuyển phản biện: 5/9/2021, ngày chấp nhận phản biện: 10/9/2021, ngày chấp nhận đăng: 15/9/021 


\section{Nghiên cúu}

trường bãi biển sạch và ảnh hưởng đến khả năng kiếm ăn của động vật hoang dã [4]. Sự tích tụ rác nhựa trên biển là một trong những nguyên nhân gây suy thoái hệ sinh thái dưới đáy biển, sinh vật sống ở tầng nước nông và hệ sinh thái ven biển [5]. Ô nhiễm nhựa và tác hại từ hóa chất trong nhựa cũng đang đe dọa nghiêm trọng đến sức khỏe của con người thông qua chuỗi thức ăn và sự suy thoái của hệ sinh thái cũng gây ảnh hưởng trực tiếp lẫn gián tiếp về kinh tế. Việc cấm sử dụng nhựa là không khả thi và không thể làm được. Thay vào đó, đưa ra một số hạn chế trong việc sử dụng, sản xuất và tiêu dùng có thể là một giải pháp hiệu quả hơn. Các biện pháp giảm thiểu - giảm nguồn ô nhiễm rác thải nhựa, sẽ yêu cầu xác định các nguồn địa lý, chẳng hạn như sông, đầm phá hoặc các địa điểm ven biển cụ thể. Ước tính trên toàn cầu, khoảng $80 \%$ nhựa đại dương đến từ đất liền và $20 \%$ còn lại từ nguồn hàng hải [6], trong đó phần lớn là rác chìm dưới đại dương và chỉ có khoảng $30 \%$ rác biển nổi trên bề mặt hoặc trong cột nước. Việc có thể phát hiện các loại nhựa trôi nổi trong vùng nước ven biển trước khi chúng bị cuốn đi, bị sinh vật biển ăn vào, hoặc phân hủy,... có thể giúp trả lời các câu hỏi về nguồn gốc, con đường đi và xu hướng của rác thải nhựa.

Trong những năm gần đây, các kỹ thuật giám sát dựa trên dữ liệu viễn thám đã được tích cực nghiên cứu để giải quyết những vấn đề này. Các phương pháp viễn thám với cảm biến thụ động (đa phổ, siêu phổ) hoặc cảm biến chủ động (lidar, sar), được kết hợp với các phương tiện trên không (máy bay không người lái) có thể là công cụ hữu ích để định lượng và giám sát sự phân bố của rác thải nhựa ven biển và trên đại dương. Phát hiện và phân loại rác thải nhựa trôi nổi trong vùng nước ven biển và gần bờ có thể được thực hiện bằng cách sử dụng nhiều thuật toán phân loại khác nhau. Tuy nhiên, các kỹ thuật này cũng có thể tốn rất nhiều thời gian nếu việc phân tích hình ảnh được thực hiện bằng thủ công [3]. Việc phát triển các thuật toán để tối ưu hóa phát hiện rác thải nhựa ven biển và trên đại dương là rất quan trọng trong việc thực hiện thành công các kỹ thuật này, bởi vì các thuật toán phân loại có thể được phát triển dựa trên tất cả hoặc một tập hợp con của dữ liệu đa phổ (quang học) hoặc giá trị tán xạ ngược (radar). Các mô hình học máy đã cho thấy kết quả tốt trong việc nhận dạng và phân loại đối tượng. Đặc biệt, các mô hình mạng nơron tích chập sâu (DCNN) đã được áp dụng rộng rãi do khả năng nhận dạng các tính năng và mẫu có trong bộ dữ liệu lớn về hình ảnh [7]. Tuy nhiên, cho đến nay không nhiều thuật toán được phát triển để phát hiện, phân loại và xác định rõ vị trí rác thải nhựa ven biển từ dữ liệu viễn thám với độ phân giải không gian cao và siêu cao. Một số tác giả đã sử dụng thông tin quang phổ để phát triển các thuật toán nhằm xây dựng mô hình tự động phát hiện các đối tượng rác thải nhựa [6], [8], [9]. Một nghiên cứu điển hình khác đã áp dụng thành công mô hình $\mathrm{CNN}$ để tự động phát hiện rác thải trên biển từ các hình ảnh được chụp ở độ cao vài mét trên mặt nước [10]. Ngoài ra, các hình ảnh cảm biến từ xa cũng được sử dụng để phát hiện rác thải nhựa đang ở giai đoạn sơ khai [11], [12]. Dù các phương pháp gần đây có cải tiến và có kết quả đáng khích lệ nhưng các thuật toán bán tự động và tự động có thể phát hiện rác thải nhựa ven biển từ ảnh viễn thám vẫn chưa được nghiên cứu nhiều. Với cách tiếp cận trên, nghiên cứu này nhằm mục đích thúc đẩy hơn nữa việc ứng dụng mô hình DCNN trong việc xác định sự hiện diện của rác thải nhựa trong môi trường ven biển và trên biển bằng cách sử dụng các hình ảnh được chụp từ thiết bị máy bay không người lái (UAV) với sự linh hoạt của thiết bị bay và thời gian chụp chủ động.

\section{Dữ liệu và phương pháp nghiên cứu}

\subsection{Xủ̉ lý dĩ liệu máy bay không người lái}

Tất cả các hình ảnh được chụp ở độ cao $350 \mathrm{~m}$ với khoảng cách lấy mẫu mặt đất nằm trong khoảng $9 \mathrm{~mm} \mathrm{pixel}^{-1}$. Tổng cộng có 95 hình ảnh với kích thước $(5472$ × 3648 pixels $)$, đã được ghi lại tại khu vực ven biển thành phố Hội An (Quảng Nam). Quá trình xử lý dữ liệu UAV được thực hiện trên phần mềm xử lý ảnh 
CATALYST PROFESSIONAL. Hình 1 minh họa sơ đồ các bước thực hiện chính: (i) Hiệu chỉnh thông số máy ảnh: bước này giúp xác định và hiệu chỉnh các biến dạng của hình ảnh do độ cong của ống kính, độ dài tiêu cự và các hiệu ứng phối cảnh; (ii) Thiết lập các điểm liên kết: điểm liên kết một vị trí, có thế được nhận trực quan trên các khu vực chồng chéo giữa hai hay nhiều hình ảnh, tổng cộng có 2.850 điểm ràng buộc tối ưu để ghép 95 hình ảnh với nhau; (iii) Tinh chỉnh điểm: trong bước này 2.850 điểm ràng buộc được thiết lập ở bước trên đã được tinh chỉnh để loại bỏ những điểm có giá trị sai số cao (những điểm có giá trị cao hơn $\pm 0,5)$ đã bị loại bỏ khỏi mô hình tính toán; (iv) Chỉnh sưa các điểm khống chế mặt đất: tổng cộng có 12 điểm GCP đã được thiết lập để hiệu chỉnh tham chiếu địa lý của tập

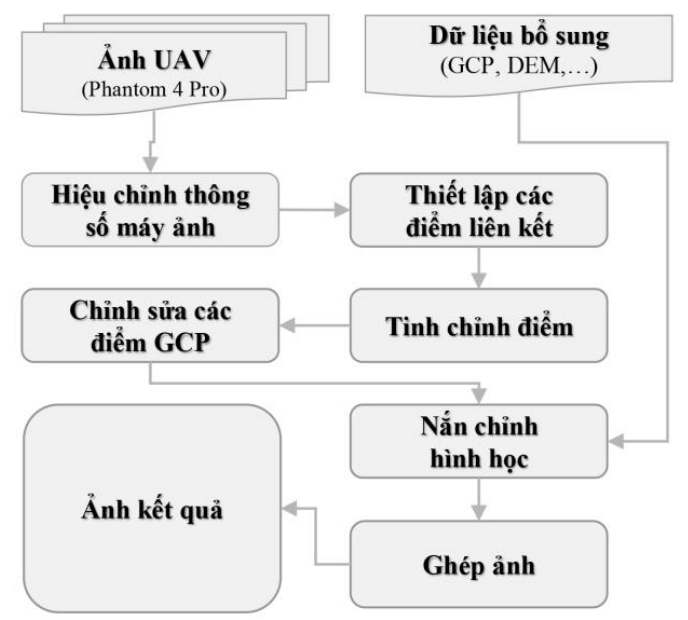

Hình 1: Quy trình các bước tiền xử lý ảnh UAV.

2.2. Mạng nơ-ron tích chập phát hiện rác thải nhụa ven biển

So với các kỹ thuật phân loại truyền thống, một thuật toán để tự động phát hiện rác thải nhựa trong ảnh UAV được phát triển bằng cách áp dụng thuật toán học sâu dựa trên kiến trúc CNN. Trong khi các thuật toán học máy khác cần một tập hợp các tính năng để cung cấp cho bộ mẫu phân loại, thì thuật toán học sâu dựa trên mô hình CNN sẽ đào tạo và nhận dạng các tập mẫu không gian của các mục tiêu bằng cách sử dụng một loạt các tính năng trực tiếp có trên hình ảnh thay vì tập trung chiết tách tính năng giống như ở các mạng nơ-ron khác. Các kiến trúc của mô hình CNN được thực hiện theo các bước chính như: dữ liệu về hệ tọa độ WGS84/UTM ZONE-48N; (v) Nắn chỉnh hình hoc sư dụng mô hình số độ $c a o$ : Ở bước này từ 95 hình ảnh thô trực quan và DEM (độ phân giải không gian $0,35 \mathrm{~m}$ ) của khu vực nghiên cứu được áp dụng. Điều này giúp nâng cao hiệu suất của quá trình tương quan và giảm xác suất kết hợp không chính xác của các hình ảnh thô ban đầu; (vi) Ghép ảnh: 95 hình ảnh trực quan đã được chỉnh sửa thủ công để tạo thành một hình ảnh duy nhất cân bằng màu sắc. Bởi vì các hình ảnh có độ phủ trùng lặp đến $80 \%$, tất cả các lỗi liên quan đến máy ảnh và tọa độ đã được loại bỏ. Sau khi các hình ảnh đơn được hợp nhất, khu vực có rác thải nhựa ven biển thành phố Hội An là đầu vào cho mô hình $\mathrm{DCNN}$ và được trình bày trong phần 2.2.

(i) Dữ liệu UAV vào mạng nơ-ron tích chập sâu, được xử lý với sự trợ giúp của các giá trị pixel của hình ảnh trong lớp tích chập; (ii) Các bộ lọc được tạo ra để thực hiện các phép lặp trên toàn bộ hình ảnh và tập huấn luyện mạng để xác định và chiết tách đối tượng từ hình ảnh $U A V$ và được chuyển đổi thành ma trận; (iii) Chuẩn hóa hàng loạt các vectơ đầu vào được thực hiện ở mỗi lớp, để đảm bảo tất cả các vectơ đầu vào được chuẩn hóa và do đó đạt được sự ổn định trong mạng; (iv) Các phép chập được thực hiện cho đến khi đạt được độ chính xác tối ưu và việc chiết tách thông tin tối đa được thực hiện; (v) Các chuyển đổi dẫn đến việc phân nhóm của hình ảnh và kích thước của đầu vào được thay đổi tùy theo vùng 


\section{Nghiên cứu}

đệm; (vi) Mỗi tích chập theo đơn vị tuyến tính chỉnh lưu (ReLU) và lớp tổng hợp; (vii) Sau tích chập cuối cùng, ma trận đầu vào được chuyển đổi thành vectơ đặc trưng. Vectơ đặc trưng này là lớp được làm phẳng; (viii) Lớp được làm phẳng đóng vai trò là đầu vào cho lớp kết nối đầy đủ, nơi tất cả các đặc trưng được chuyển chung vào mạng này. Bỏ qua các nút ngẫu nhiên xảy ra trong quá trình đào tạo để giảm tần suất quá mức trong lớp này; (ix) Cuối cùng, các giá trị thô được dự đoán đầu ra bởi mạng nơ-ron được chuyển đổi thành giá trị xác suất bằng cách sử dụng hàm softmax. Mô hình $\mathrm{CNN}$ trong môi trường học sâu được đào tạo trước để phân loại tập hợp thử nghiệm các hình ảnh trong một loạt 2 lớp được xác định trước (Hình 2). Do đó, khi các hình ảnh được chia thành nhiều ô, thuật toán sẽ phân loại từng ô của các hình ảnh có chứa rác thải nhựa hay không chứa rác thải nhựa (Hình 3).

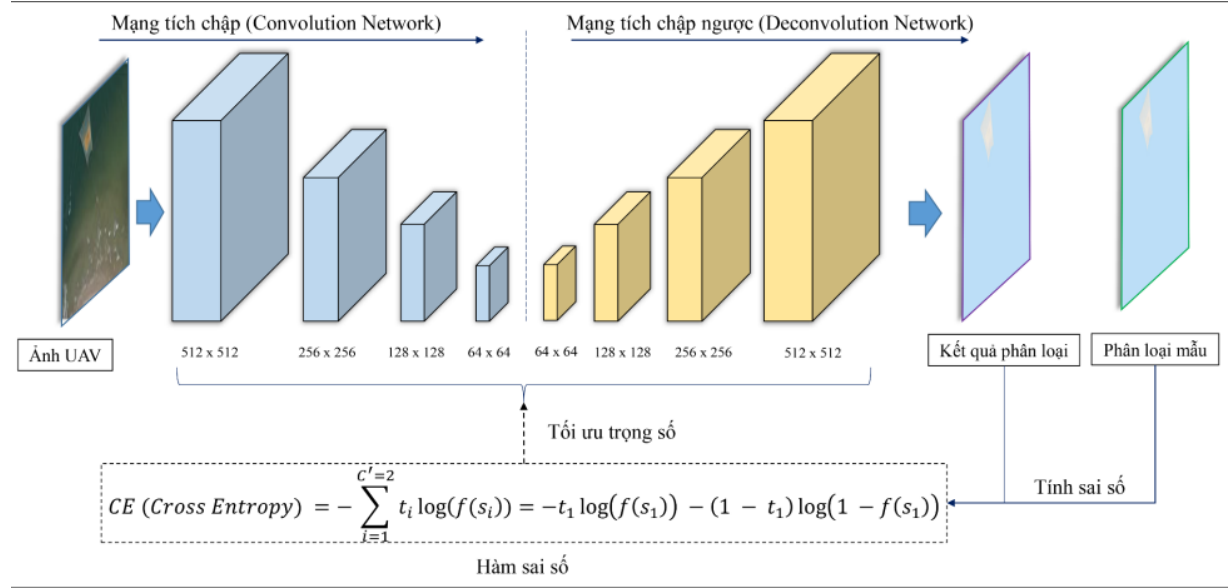

Hình 2: Mô hình phân loại theo pixel cho việc xác định rác thải nhựa

Hàm sai số Cross Entropy $(\mathrm{CE})$ được xác định bởi công thức 1 :

$$
C E=-\sum_{i=1}^{C^{\prime}=2} t_{i} \log \left(f\left(s_{i}\right)\right)=-t_{1} \log \left(f\left(s_{1}\right)\right)-\left(1-t_{1}\right) \log \left(1-f\left(s_{1}\right)\right)
$$

Với phân loại nhị phân (binary sẽ đi qua hàm $f()$ là hàm Sigmoid $f\left(s_{i}\right)=$ segmentation) cho hai lớp $C_{1}$ và $C_{2}$ tương ứng cho "có rác thải nhựa" và "không có rác thải nhựa". $t_{1}[0,1]$ và $s_{1}$ lần lượt là phân loại mẫu và điểm số cho lớp $C_{1}, t_{2}=1-t_{1}$ và $s_{2}=1-$ $s_{1}$ là phân loại mẫu và điểm số cho lớp $C_{2}$. Trong phép phân loại nhị phân này, kết quả của mô hình

$\frac{1}{1+e^{-s_{i}}}$ để đưa kết quả dự đoán về khoảng $[0,1]$.

Các phép toán tích chập và phân lớp được thực hiện theo công thức 2 , các ma trận kết quả của các lớp tích chập (Conv) đã được làm phẳng. Công thức 3 đại điện cho mô hình hoàn chỉnh bao gồm các lớp được kết nối đầy đủ (Hình 3).

$$
\begin{gathered}
\operatorname{Conv}=P\left[a\left[\sum_{q=1}^{128} a\left(\sum_{q=1}^{128}\left(P\left[a\left(\sum_{q=1}^{64} I^{*} K_{1, q}^{1}+b_{q}^{2}\right)\right]_{\max (2 i, 2 j)}\right) * K_{1, q}^{3}+b_{q}^{3}\right) * K_{1, q}^{4}+b_{q}^{4}\right)\right] \\
\hat{Y}=\operatorname{soft} \max \left[\sum_{q=1}^{500}\left[a\left(\sum_{q=1}^{1600} C_{a} \times K_{1, q}^{5}+b_{q}^{5}\right)\right] \times K_{1, q}^{6}+b_{q}^{6}\right]
\end{gathered}
$$

Trong đó: $K_{p, q}^{l}$ là ma trận chập; $l$ là số lớp; $p \quad \hat{Y}$ là đầu ra (rác thải nhựa/không phải rác thải

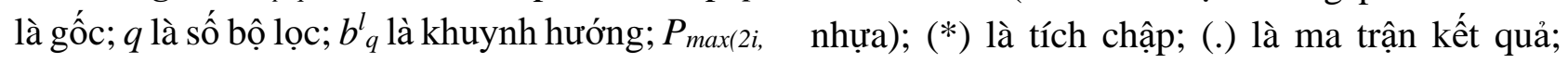
2j) là phân nhóm; $a$ là kích hoạt chức năng ReLU; $\quad C o n v$ là quá trình tích chập và phân nhóm. 


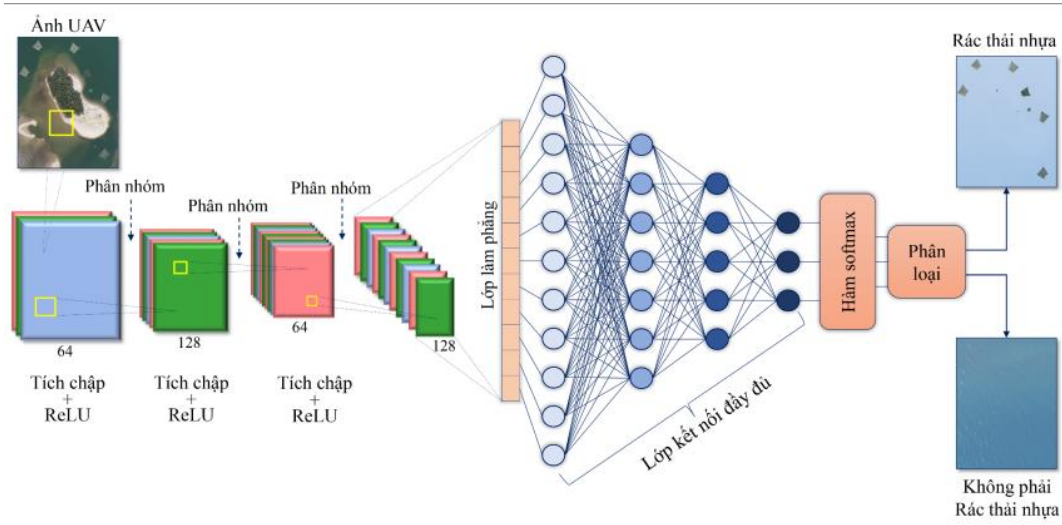

Hình 3: Quy trình học sâu của mạng no-ron tích chập trong xủ lý hình ảnh chiết tách rác thải nhựa tù ảnh UAV (máy bay không ngườ lái). ReLU (đơn vị tuyến tính chỉnh lưu)

\section{3. Đánh giá độ chính xác}

Khu vực nghiên cứu có tổng cộng 95 hình ảnh được chụp (50 hình ảnh có rác thải nhựa và 45 hình ảnh không có rác thải nhựa) được sử dụng để đào tạo/ kiểm chứng mô hình trong quá trình phân loại $80 \%$ và $20 \%$ trong số đó được sử dụng để mẫu đào tạo và kiểm chứng mô hình, tương ứng trong quá trình xác nhận chéo. Độ chính xác tổng thể $(\mathrm{OA})$ của phương pháp học sâu dựa trên mô hình $\mathrm{CNN}$ được tính theo công thức (4), phân loại chính xác các hình ảnh có hoặc không có rác thải nhựa, và xác nhận chéo (CV) được tính theo công thức (5), phân loại là có rác thải nhựa thực sự thuộc về lớp đó.

$$
\begin{array}{r}
O A=\frac{T P+T N}{T N+T P+F N+F P} \\
C V=\frac{\frac{2 \times T P}{(T P+F N)} \times \frac{T P}{(T P+F P)}}{\frac{T P}{(T P+F N)}+\frac{T P}{(T P+F P)}}
\end{array}
$$

Trong đó: $T P$ là đối tượng có rác thải nhựa phân loại đúng; $T N$ là đối tượng không có rác thải nhựa phân loại đúng; $F P$ là đối tượng rác thải nhựa phân loại sai; và $F N$ là đối tượng không có rác thải nhựa phân loại sai. Quá trình xử lý và tính toán theo thuật toán học sâu trên mô hình CNN được thiết lập dựa trên bộ máy trạm với cấu hình RAM 64GB Bus $3200 \mathrm{MHz}$ và card đồ họa NVIDIA GeForce RTX 2080 Max-Q.

\section{Kết quả và thảo luận}

\section{1. Đánh giá mô hình học sâu dụa trên} mạng no-ron tích chập

Độ chính xác của mô hình nghiên cứu đã được kiểm chứng trong quá trình phân loại và xác nhận chéo. Tất cả các hình ảnh được gắn nhãn và được sử dụng để đánh giá độ chính xác tổng thể gồm 50 hình ảnh có rác thải nhựa và 45 hình ảnh không có rác thải nhựa. Độ chính xác tổng thể $(\mathrm{OA})$ của kết quả phân loại đạt 0,87 và kết quả xác nhận chéo $(\mathrm{CV})$ đạt 0,83 . Độ chính xác của $\mathrm{CV}$ thấp hơn so với $\mathrm{OA}$, bởi vì quy trình đầu tiên trong thuật toán học sâu dựa trên mô hình $\mathrm{CNN}$ sử dụng hình ảnh khác nhau để đào tạo và kiểm chứng, trong khi các hình ảnh giống nhau được sử dụng để đào tạo và kiểm chứng trong quá trình phân loại. Mức độ lặp lại của phương pháp kiểm chứng được xử lý 10 lần các bộ ảnh được chọn ngẫu nhiên (80\% số mẫu được sử dụng để đào tạo và $20 \%$ số mẫu được sử dụng để kiểm chứng kết quả mô hình) được tính toán cho mỗi bộ dữ liệu.

Trong nghiên cứu này, nhóm tác giả đã tập trung áp dụng thuật toán học sâu dựa trên mạng nơ-ron tích chập (DCNN) để phát hiện và chiết tách rác thải nhựa từ các hình ảnh được chụp bằng máy bay không người lái (UAV). Kết quả thu được từ việc áp dụng mô hình học sâu $\mathrm{CNN}$ tối ưu để phân tích 95 hình ảnh UAV đạt độ chính xác tốt cho việc chiết tách rác thải nhựa ven biển. Kích thước, hình dạng và màu sắc của vật thể là các đặc điểm quan trọng được sử dụng trong các thuật toán định lượng, nhận dạng hoặc 


\section{Nghiên cúu}

phát hiện [13]. Kết quả phát hiện rác thải nhựa khu vực ven biển thành phố Hội An được minh họa một phần trong Hình 4. Trong đó, (i) Đối tượng một tấm lưới đánh cá có kích thước lớn, có kết cấu được làm từ vật liệu nhựa và chất liệu dẻo (Hình 4a); (ii) Các tấm lưới đánh cá được phân bố rải rác và được giữ bằng kết cấu khung có hình dạng nhất định, độ phản xạ quang phổ của lưới đánh cá không đồng nhất, đó có thể là các tấm lưới bẫy rác thải nhựa làm từ ngư cụ cũ được sử dụng ven khu vực nuôi trồng thủy sản nhằm cản lại lượng rác thải nhựa và lọc môi trường xung quanh lồng bè (Hình $4 \mathrm{~b}$ ); (iii) Đối tượng các tấm xốp có màu trắng, bề mặt có khả năng bị nhiễm bẩn trong quá trình nổi ở mực nước biển, có thể gây khó khăn trong việc phát hiện khi sử dụng màu sắc hoặc độ phản xạ, tuy nhiên, khi sử dụng mô hình DCNN có thể phát hiện tốt xốp, ngay cả khi rác thải xốp ở gần bãi cát ven bờ biển đã bị đổi màu (Hình $4 \mathrm{c}$ ); (iv) Đối tượng các mảnh rác thải nhựa ở các kích thước khác nhau (chai nhựa, túi nhựa, cốc nhựa, thùng nhựa...) trên bãi cát ven biển, mô hình cũng phát hiện và chiết tách (Hình 4d). Tuy nhiên, vẫn còn tồn tại một số khó khăn do sự đa dạng về điểm nhìn, vật thể ẩn sau đối tượng và sự đa dạng chủng loại rác thải nhựa, nên mô hình học sâu DCNN vẫn nhận dạng chưa được chính xác một số đối tượng là các rác thải khác như mảnh gỗ lẫn với các mảnh rác thải nhựa. Việc phát triển thuật toán hoàn thiện cần phải có các phép đo chất lượng cao từ các mục tiêu khác nhau để giảm thiểu nhầm lẫn trong các ứng dụng giám sát trong tương lai liên quan đến vật liệu nhựa.
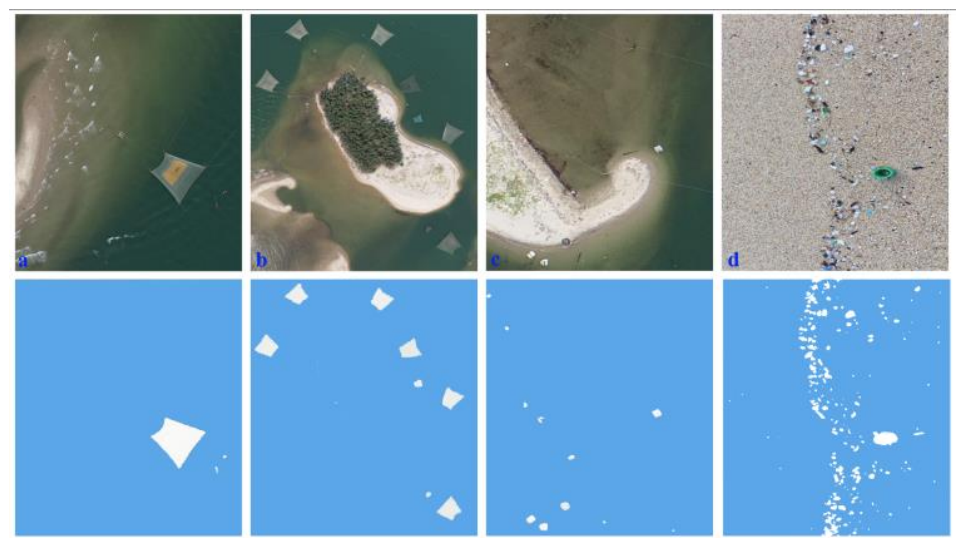

Hình 4: Kết quả phát hiện rác thải nhựa tù mạng no-ron tích chập sâu (phía trên: ảnh đầu vào; phía dưới: kết quả chiết tách rác thải nhựa)

\subsection{Thảo luận}

Tình trạng ô nhiễm rác thải nhựa ven biển hay "ô nhiễm trắng" được xem là thách thức lớn đối với môi trường bởi khối lượng cũng như số lượng của rác thải nhựa. Nguồn gây ô nhiễm chính là nguồn thải trên đất liền và các nguồn thải trên biển, đóng góp chính là rác thải nhựa có kích thước lớn, bao gồm các vật dụng hàng ngày và các loại bao bì đóng gói khác. Phần còn lại là nhựa được xả trực tiếp trên biển, chủ yếu là từ hoạt động khai thác thủy sản. Việc thải ra môi trường rác thải nhựa không đúng cách vào đại dương và đất liền sẽ gây nguy hiểm cho các loài sinh vật biển và ảnh hưởng đến sức khỏe cũng như tính mạng con người. Ngoài ra, vấn đề ô nhiễm rác thải nhựa đã và đang gây ra các tác động tiêu cực đến kinh tế - xã hội dựa trên nhiều khía cạnh khác nhau như thương mại, du lịch, vận tải biển... và ảnh hưởng đến nền kinh tế quốc dân của quốc gia bằng cách chi viện thêm ngân sách cho việc xử lý rác thải nhựa. Đặc biệt, trong tình hình căng thẳng của đại dịch Covid-19 lượng rác thải y tế tăng đột biến đang là mối lo ngại đối với môi trường ven biển và sức khỏe của con người [14]. Sự gia tăng đáng kể trong việc sử dụng khẩu trang $\mathrm{y}$ tế, găng tay và quần áo bảo hộ, ống tiêm... phục vụ cho công tác phòng chống đại dịch tất cả đều đang góp phần gây ra tình trạng ô nhiễm rác thải nhựa này. Một nghiên cứu đã chỉ ra rằng các sản phẩm y tế có thể gây 
ra 44,8\% và $13,2 \%$ sự gia tăng chất thải nhựa do đại dịch hiện nay [15], [16]. Vì lý do này, việc thúc đẩy điều tra, khảo sát đánh giá hiện trạng rác thải nhựa và hoàn thiện chính sách, quy định về quản lý rác thải nhựa; nghiên cứu, ứng dụng công nghệ, triển khai mô hình, hoạt động quản lý rác thải nhựa và sản xuất các sản phẩm thân thiện với môi trường là cấp thiết trong bối cảnh hiện nay.

Kết quả của nghiên cứu hỗ trợ thêm cho việc sử dụng các cảm biến trên không để kiểm tra bề mặt biển và phát hiện rác thải nhựa. Dựa trên thuật toán học máy nâng cao mở ra tiềm năng ứng dụng kỹ thuật và công nghệ tự động trong việc phát hiện rác thải nhựa nổi trên biển ở quy mô lớn bằng dữ liệu và công nghệ viễn thám tại Việt Nam. Các nghiên cứu dựa trên kỹ thuật học sâu CNN để phát hiện và theo dõi rác thải nhựa đã được các học giả quan tâm đáng kể trong những năm gần đây. Ảnh UAV đã được sử dụng cho các mục đích này ở quy mô lớn, chẳng hạn như để giám sát các bãi rác lớn dọc theo bờ biển [17], [18]. Tuy nhiên, những nghiên cứu của các học giả đi trước sử dụng mô hình $\mathrm{CNN}$ thường hay gặp phải chiết tách rác thải nhựa ven biển bị lẫn với gỗ tự nhiên và thảm thực vật trên cồn cát. Các thuật toán học sâu $\mathrm{CNN}$ được trình bày trong nghiên cứu hiện tại đã được điều chỉnh từ những thuật toán hiện có với mục tiêu hợp lý hóa quá trình phát hiện và chiết tách rác thải nhựa trong các hình ảnh được chụp từ thiết bị bay chụp không người lái (UAV), có thể hữu ích khi phân loại số lượng lớn hình ảnh có hay không có rác thải nhựa, do đó có thể giúp xác định các khu vực tiềm ẩn nguy cơ tập hợp rác ven biển và trên biển. Viễn thám nghiên cứu rác thải nhựa ven biển và trên đại dương là một thách thức về phương diện công nghệ vẫn không ngừng được nghiên cứu và phát triển. Tuy nhiên, một thách thức quan trọng khác đối với các nỗ lực giám sát rác thải nhựa ven biển là việc xin giấy phép bay chụp ở các khu vực quan tâm khác nhau. Các nghiên cứu đang thực hiện và trong tương lai cần xem xét việc phát triển các thuật toán học sâu để xác định kích thước, thông tin quang phổ và loại rác thải nhựa, đây là những thông tin hữu ích có liên quan để các nhà hoạch định chính sách đưa ra các giải pháp nhằm giảm thiểu nguy cơ rác thải nhựa và bảo vệ môi trường được tốt hơn.

\section{Kết luận}

Trong bài báo này, nhóm tác giả đề xuất việc sử dụng mô hình mạng nơ-ron tích chập sâu (DCNN) để xử lý các hình ảnh UAV nhằm phát hiện tự động rác thải nhựa ven biển. CNN có cấu trúc đặc biệt là các lớp tích chập và các phân nhóm để chiết tách các tính năng khác nhau của dữ liệu. Ngoài ra, khi nhiều lớp tích chập và phân nhóm được sử dụng, có thể chiết tách tính năng toàn cục một lần nữa từ tính năng toàn cục được chiết tách trong quá trình trước đó. Các đặc điểm của mô hình CNN có thể hữu ích cho việc xác định rác thải nhựa ven biển. Việc nghiên cứu sâu hơn là cần thiết để cải thiện các thuật toán tự động hiện tại bằng cách tăng số lượng hình ảnh được sử dụng để huấn luyện, cải thiện độ chính xác của mô hình. Các phương pháp tự động phát hiện và chiết tách rác thải nhựa có thể bổ sung hoặc thay thế các phương pháp truyền thống để giám sát môi trường biển và cải thiện đáng kể chất lượng kết quả phân loại. Khám phá cơ hội sử dụng dữ liệu viễn thám có độ phân giải cao hơn, đa góc độ, có sẵn trên thị trường để phát triển một thuật toán phân loại tốt ưu. $\bigcirc$

\section{Lời cảm ơn}

Nghiên cứu này được tài trợ bởi đề tài "Nghiên cứu xây dựng công nghệ điều tra, giám sát và bản đồ phân vùng rủi ro ô nhiễm rác thải nhựa ở biển Việt Nam", mã số ĐTĐLCN.55/20.

\section{Tài liệu tham khảo}

[1]. A. C. Ciappa, "Marine plastic litter detection offshore Hawai'i by Sentinel-2," Mar. Pollut. Bull., vol. 168, p. 112457, Jul. 2021, doi: 10.1016/j.marpolbul.2021.112457.

[2]. A. Melet et al., "Earth Observations for Monitoring Marine Coastal Hazards and Their Drivers," Surv. Geophys., vol. 41, no. 6, pp. 


\section{Nghiên cứu}

1489-1534, Nov. 2020, doi: 10.1007/s 10712020-09594-5.

[3]. V. Martínez-Vicente et al., "Measuring Marine Plastic Debris from Space: Initial Assessment of Observation Requirements," Remote Sens., vol. 11, no. 20, p. 2443, Oct. 2019, doi: 10.3390/rs11202443.

[4]. S. Ghaffari, A. R. Bakhtiari, S. M. Ghasempouri, and A. Nasrolahi, "The influence of human activity and morphological characteristics of beaches on plastic debris distribution along the Caspian Sea as a closed water body," Environ. Sci. Pollut. Res., vol. 26, no. 25, pp. 25712-25724, Sep. 2019, doi: 10.1007/s11356-019-05790-y.

[5]. M. Thiel et al., "Impacts of Marine Plastic Pollution From Continental Coasts to Subtropical Gyres-Fish, Seabirds, and Other Vertebrates in the SE Pacific," Front. Mar. Sci., vol. 5, p. 238, Jul. 2018, doi: 10.3389/fmars.2018.00238.

[6]. K. Themistocleous, C. Papoutsa, S. Michaelides, and D. Hadjimitsis, "Investigating Detection of Floating Plastic Litter from Space Using Sentinel-2 Imagery,”p. 18, 2020.

[7]. I. Marin, S. Mladenović, S. Gotovac, and G. Zaharija, "Deep-Feature-Based Approach to Marine Debris Classification," Appl. Sci., vol. 11, no. 12, p. 5644, Jun. 2021, doi: 10.3390/app11125644.

[8]. S. H. Bak, D. H. Hwang, H. M. Kim, and H. J. Yoon, "DETECTION AND MONITORING OF BEACH LITTERUSING UAV IMAGE AND DEEP NEURAL NETWORK," Int. Arch. Photogramm. Remote Sens. Spat. Inf. Sci., vol. XLII-3/W8, pp. 55-58, Aug. 2019, doi: 10.5194/isprs-archives-XLII-3W8-55-2019.

[9]. P. Tasseron, T. van Emmerik, J. Peller, L. Schreyers, and L. Biermann, "Advancing Floating Macroplastic Detection from Space Using Experimental Hyperspectral Imagery,"
Remote Sens., vol. 13, no. 12, p. 2335, Jun. 2021, doi: $10.3390 /$ rs 13122335 .

[10]. G. Gonçalves, U. Andriolo, L. Pinto, and F. Bessa, "Mapping marine litter using UAS on a beach-dune system: a multidisciplinary approach," Sci. Total Environ., vol. 706, p. 135742, Mar. 2020, doi: 10.1016/j.scitotenv.2019.135742.

[11]. K. Topouzelis, D. Papageorgiou, A. Karagaitanakis, A. Papakonstantinou, and M. Arias Ballesteros, "Remote Sensing of Sea Surface Artificial Floating Plastic Targets with Sentinel-2 and Unmanned Aerial Systems (Plastic Litter Project 2019)," Remote Sens., vol. 12, no. 12, p. 2013, Jun. 2020, doi: 10.3390/rs12122013.

[12]. C. N. Koyama, H. Gokon, M. Jimbo, S. Koshimura, and M. Sato, "Disaster debris estimation using high-resolution polarimetric stereo-SAR," ISPRS J. Photogramm. Remote Sens., vol. 120, pp. 84-98, Oct. 2016, doi: 10.1016/j.isprsjprs.2016.08.003.

[13]. Z.-Q. Zhao, P. Zheng, S. Xu, and X. Wu, "Object Detection with Deep Learning: A Review," ArXiv180705511 Cs, Apr. 2019, Accessed: Oct. 31, 2021. [Online]. Available: http://arxiv.org/abs/1807.05511

[14]. N. U. Benson, D. E. Bassey, and T. Palanisami, "COVID pollution: impact of COVID-19 pandemic on global plastic waste footprint," Heliyon, vol. 7, no. 2, p. e06343, Feb. 2021, doi: 10.1016/j.heliyon.2021.e06343.

[15]. M. Shams, I. Alam, and M. S. Mahbub, "Plastic pollution during COVID-19: Plastic waste directives and its long-term impact on the environment," Environ. Adv., vol. 5, p. 100119, Oct. 2021, doi: 10.1016/j.envadv.2021.100119.

[16]. H. B. Sharma et al., "Challenges, opportunities, and innovations for effective solid waste management during and post COVID-19 pandemic," Resour. Conserv. Recycl., vol. 162, p. 105052, Nov. 2020, doi: 10.1016/j.resconrec.2020.105052. 
[17]. G. Gonçalves, U. Andriolo, L. Pinto, and D. Duarte, "Mapping marine litter with Unmanned Aerial Systems: A showcase comparison among manual image screening and machine learning techniques," Mar. Pollut. Bull., vol. 155, p. 111158, Jun. 2020, doi: 10.1016/j.marpolbul.2020.111158.

[18]. G. Jakovljevic, M. Govedarica, and F. Alvarez-Taboada, “A Deep Learning Model for

\section{Summary}

Study model for detection on coastal plastic waste using unmanned aerial vehicle image and deep convolutional neural network

Do Thi Nhung, Nguyen Thi Diem My, Pham Van Manh, Pham Vu Dong, Bui Quang Thanh

University of Science, Vietnam National University, Hanoi

Nghiem Van Tuan

Department of National Remote Sensing, Ministry of Natural Resources and Environment

\section{Pham Minh Hai}

Vietnam Institute of Geodesy and Cartography, Ministry of Natural Resources and Environment

Plastic waste in coastal areas originating from human activities is becoming a danger to the marine environment because of its large quantity and difficulty to decompose. Consequently, they have damaged drastically marine ecosystems as well as the above-ground environments. Plastic wastes monitoring and prevention programs have been brought into focus over the world and supported by modern technologies. Unmanned Aerial vehicles (UAV) and Deep Convolutional neural networks (DCNN) have been well known for being highly effective for monitoring, detecting, and classifying plastic waste in coastal areas. This study proposes an algorithm for detecting plastic wastes by exploiting DCNN that was trained with labeled spatial data. The deep network was trained and tested with 95 images from Phantom 4 Pro UAV with CMOS camera-equipped flying over Hoi An coastal (Quang Nam province). The results that our network can detect plastic wastes with precision and cross-validation were 0.87 and 0.83 , respectively. To conclude, this study provides a new approach for researchers and authorities to extend the UAV image source for monitoring and evaluating the impacts of plastic wastes on coastal areas. Nevertheless, automatic monitoring has still remained a challenging task and needs to be further studied with modern algorithms in the future. $\bigcirc$

Keywords: Plastic waste, Remote sensing, UAV, Convolutional neural network, Deep learning. 